\title{
FRESHLY FORMED DUST IN THE CASSIOPEIA A SUPERNOVA REMNANT AS REVEALED BY THE SPITZER SPACE TELESCOPE
}

\author{
J. Rho, ${ }^{1}$ T. Kozasa, ${ }^{2}$ W. T. Reach, ${ }^{1}$ J. D. Smith, ${ }^{3}$ L. Rudnick, ${ }^{4}$ T. DeLaney, ${ }^{5}$ \\ J. A. EnNis, ${ }^{4}$ H. Gomez, ${ }^{6}$ And A. TAPPE ${ }^{1,7}$ \\ Received 2007 March 20; accepted 2007 September 4
}

\begin{abstract}
We performed Spitzer Infrared Spectrograph mapping observations covering nearly the entire extent of the Cassiopeia A supernova remnant (SNR), producing mid-infrared (5.5-35 $\mu \mathrm{m}$ ) spectra every $5^{\prime \prime}-10^{\prime \prime}$. Gas lines of Ar, $\mathrm{Ne}, \mathrm{O}$, $\mathrm{Si}, \mathrm{S}$, and $\mathrm{Fe}$, and dust continua were strong for most positions. We identify three distinct ejecta dust populations based on their continuum shapes. The dominant dust continuum shape exhibits a strong peak at $21 \mu \mathrm{m}$. A line-free map of $21 \mu \mathrm{m}$ peak dust made from the 19-23 $\mu \mathrm{m}$ range closely resembles the [Ar II], [O IV], and [ $\mathrm{Ne}$ II] ejecta-line maps, implying that dust is freshly formed in the ejecta. Spectral fitting implies the presence of $\mathrm{SiO}_{2}, \mathrm{Mg}$ protosilicates, and $\mathrm{FeO}$ grains in these regions. The second dust type exhibits a rising continuum up to $21 \mu \mathrm{m}$ and then flattens thereafter. This "weak $21 \mu \mathrm{m}$ " dust is likely composed of $\mathrm{Al}_{2} \mathrm{O}_{3}$ and $\mathrm{C}$ grains. The third dust continuum shape is featureless with a gently rising spectrum and is likely composed of $\mathrm{MgSiO}_{3}$ and either $\mathrm{Al}_{2} \mathrm{O}_{3}$ or Fe grains. Using the least massive composition for each of the three dust classes yields a total mass of $0.020 M_{\odot}$. Using the most massive composition yields a total mass of $0.054 M_{\odot}$. The primary uncertainty in the total dust mass stems from the selection of the dust composition necessary for fitting the featureless dust as well as $70 \mu \mathrm{m}$ flux. The freshly formed dust mass derived from Cas A is sufficient from SNe to explain the lower limit on the dust masses in high-redshift galaxies.
\end{abstract}

Subject headings: dust, extinction — supernovae: general — supernova remnants

\section{INTRODUCTION}

The recent discovery of huge quantities of dust $\left(10^{8}-10^{9} M_{\odot}\right)$ in very high redshifted galaxies and quasars ( Isaak et al. 2002; Bertoldi et al. 2003) suggests that dust was produced efficiently in the first generation of supernovae ( $\mathrm{SNe}$ ). Theoretical studies (Kozasa et al. 1991; Todini \& Ferrara 2001, hereafter TF01; Nozawa et al. 2003, hereafter N03) predicted the formation of a significant quantity of dust $\left(\sim 0.1-1.0 M_{\odot}\right)$ in the ejecta of Type II $\mathrm{SNe}$, and the predicted dust mass is believed to be sufficient to account for the quantity of dust observed at high redshifts (Maiolino et al. 2006; Meikle et al. 2007). Recently, a model of dust evolution in high-redshift galaxies (Dwek et al. 2007) indicates that at least $1 M_{\odot}$ of dust per SN is necessary for reproducing the observed dust mass in one hyperluminous quasar at $z=6.4$. Observationally, the presence of freshly formed dust has been confirmed in a few corecollapsed SNe, such as SN 1987A, which clearly showed several signs of dust formation in the ejecta (see McCray 1993 for details). The highest dust mass obtained so far for SN 1987A is $7.5 \times$ $10^{-4} M_{\odot}$ (Ercolano et al. 2007). Recent Spitzer and HST observations (Sugerman et al. 2006) showed that up to $0.02 M_{\odot}$ of dust formed in the ejecta of SN 2003gd with the progenitor mass of 6-12 $M_{\odot}$, and the authors concluded that SNe are major dust factories. However, from the detailed analysis of the latetime mid-infrared observations, Meikle et al. (2007) found that

\footnotetext{
1 Spitzer Science Center, California Institute of Technology, Pasadena, CA 91125; rho@ipac.caltech.edu, reach@ipac.caltech.edu.

2 Department of Cosmosciences, Graduate School of Science, Hokkaido University, Sapporo 060-0810, Japan.

3 Steward Observatory, 933 North Cherry Avenue, Tucson, AZ 85712.

4 Department of Astronomy, University of Minnesota, 116 Church Street SE, Minneapolis, MN 55455.

5 MIT Kavli Institute, 77 Massachusetts Avenue, Room NE80-6079, Cambridge, MA 02139.

6 School of Physics and Astronomy, University of Wales, Cardiff, Wales, UK.

7 Harvard-Smithsonian Center for Astrophysics, 60 Garden Street, Cambridge, MA 02138.
}

the mass of freshly formed dust in the same SN is only $4 \times$ $10^{-5} M_{\odot}$ and failed to confirm the presence of $0.02 M_{\odot}$ of dust in the ejecta. The total amount of dust that condenses in corecollapse $\mathrm{SNe}$ remains uncertain and will benefit from further investigation.

Cassiopeia A (Cas A) is the only Galactic supernova remnant (SNR) that exhibits clear evidence of dust formed in ejecta (Lagage et al. 1996; Arendt et al. 1999, hereafter ADM99). The amount of dust that forms in the ejecta of young SNRs is still controversial. Previous observations inferred only $<3 \times 10^{-3} M_{\odot}$ of dust at temperatures between 90 and 350 K (ADM99; Douvion et al. 2001, hereafter D01); this estimate is $2-3$ orders of magnitude too little to explain the dust observed in the early universe. Recent submillimeter observations of Cas A and Kepler with SCUBA (Dunne et al. 2003; Morgan et al. 2003) revealed the presence of large amounts of cold dust $\left(\sim 0.3-2 M_{\odot}\right.$ at $15-$ $20 \mathrm{~K}$ ) missed by previous IRAS/ISO observations. On the other hand, highly elongated conductive needles with mass of only $10^{-4}$ to $10^{-3} M_{\odot}$ could also explain a high submillimeter flux of Cas A, when including grain destruction by sputtering (Dwek 2004), although the physicality of such needles is doubtful (Gomez et al. 2005). While Krause et al. (2004) showed that much of the $160 \mu \mathrm{m}$ emission observed with the Multiband Imaging Photometer for Spitzer (MIPS) is foreground material, suggesting that there is no cold dust in Cas A, Wilson \& Batrla (2005) used CO emission toward the remnant to show that up to about a solar mass of dust could still be associated with the ejecta, not with the foreground material. These controversial scenarios of dust mass highlight the importance of correctly identifying the features and masses of dust freshly formed in Cas A. The Galactic young SNR Cas A allows us to study in detail the distribution and the compositions of the dust relative to the ejecta and forward shock with the Infrared Spectrograph (IRS) on board the Spitzer Space Telescope.

Cas A is one of the youngest Galactic SNRs, with an age of $335 \mathrm{yr}$ attributed to a SN explosion in AD 1671. The progenitor of Cas A is believed to be a Wolf-Rayet star with high nitrogen 


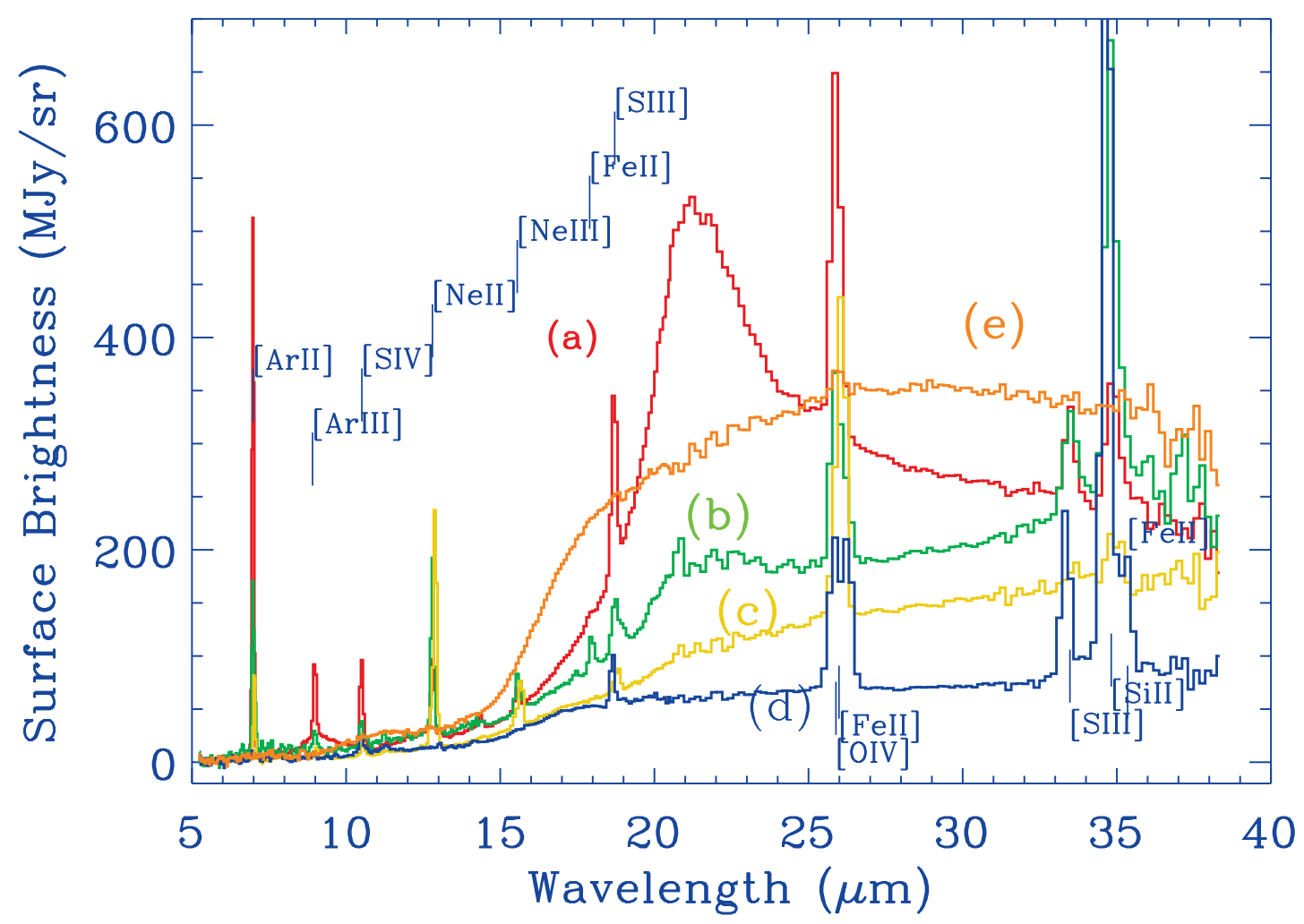

FIG. 1.-Representative set of Spitzer IRS spectra of Cas A. The contrast between continuum shape and line emission is noticeable. Many of the strong ejecta lines are

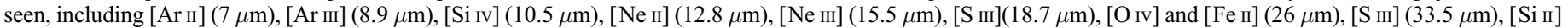
$(34.8 \mu \mathrm{m})$, and $[\mathrm{Fe} \mathrm{II}](35.3 \mu \mathrm{m})$. The dominant continuum shapes are $21 \mu \mathrm{m}$ peak dust showing a dust feature at $21 \mu \mathrm{m}$ often accompanied by a silicate emission feature at $9.8 \mu \mathrm{m}$ with strong Ar lines (red, curve a) and weak $21 \mu \mathrm{m}$ dust with relatively strong Ne lines compared with Ar (green, curve b). Featureless spectra include the continuous rising spectra ( yellow, curve c), and the gently rising spectra (blue, curve d) with strong $\mathrm{O}+\mathrm{Fe}$ or Si lines. The positions of R.A. and decl. are $350.900,58.8356$ (curve a), 350.812, 58.8075 (b), 350.879 58.7911 (c), 350.857, 58.815 (d), and 350.862 58.85509 (e). For illustration, the spectra were multiplied by 1.4 (curve a), 2.8 (b), 0.8 (c), $1.5(\mathrm{~d})$, and 2 (e), respectively. "Broad" continuum spectra arise from interstellar/circumstellar medium (orange, curve e).

abundance (Fesen 2001) and to have a mass of 15-25 $M_{\odot}$ (Kifonidis et al. 2000; Young et al. 2006) or 29-30 $M_{\odot}$ (PérezRendón et al. 2002). The predicted dust mass formed in $\mathrm{SNe}$ depends on the progenitor mass; for a progenitor mass of $15-$ $30 M_{\odot}$, the predicted dust mass is from 0.3 to $1.1 M_{\odot}(\mathrm{N} 03)$ and from 0.08 to $1.0 M_{\odot}$ (TF01), respectively. In this paper, we present Spitzer IRS mapping observations of Cas A, identify three distinct classes of dust associated with the ejecta, and discuss dust formation and composition with an estimate of the total mass of freshly formed dust.

\section{THE IRS SPECTRA AND DUST MAPS}

We performed Spitzer IRS mapping observations covering nearly the entire extent of Cas A on 2005 January 13 with a total exposure time of $11.3 \mathrm{hr}$. The Short-Low (SL: 5-15 $\mu \mathrm{m}$ ) and LongLow (LL: $15-40 \mu \mathrm{m}$ ) IRS mapping involved $\sim 16 \times 360$ and $4 \times 91$ pointings, producing spectra every $5^{\prime \prime}$ and $10^{\prime \prime}$, respectively. The spectra were processed with the S12 version of the IRS pipeline using the CUBISM package (Kennicutt et al. 2003; Smith et al. 2007), whereby backgrounds were subtracted and an extended emission correction was applied. The spectral resolving power of the IRS SL and LL modules ranges from 62 to 124 .

The IRS spectra of Cas A show bright ejecta emission lines from $\mathrm{Ar}, \mathrm{Ne}, \mathrm{S}, \mathrm{Si}, \mathrm{O}$, and $\mathrm{Fe}$ and various continuum shapes as indicated by the representative spectra in Figure 1. The most common continuum shape exhibits a large bump peaking at $21 \mu \mathrm{m}$ as shown by spectrum "a" in Figure 1. This " $21 \mu \mathrm{m}$ peak" dust is often accompanied by the silicate emission feature at $9.8 \mu \mathrm{m}$, which corresponds to the stretching mode. A second class of continuum shapes exhibits a rather sharp rise up to $21 \mu \mathrm{m}$ and then stays flat thereafter. This "weak $21 \mu \mathrm{m}$ dust" is often associated with relatively strong Ne lines (in comparison with Ar lines) and is indicated by spectrum " $b$ " in Figure 1. The third type of dust continuum is characterized by a smooth and featureless, gently rising spectrum with strong $\left[\mathrm{O}_{\mathrm{IV}}\right]+\left[\mathrm{Fe}_{\mathrm{II}}\right]$ and $[\mathrm{Si} \mathrm{II}]$ emission lines as shown by spectra "c" and "d" in Figure 1. Spectrum d shows double line structures that may be due to Doppler-resolved lines of [O IV] at $26 \mu \mathrm{m}$ and [Si II] at $35 \mu \mathrm{m}$. Note that the "featureless" dust (spectrum $d$ in Fig. 1) is a class of dust, separate from the interstellar/ circumstellar dust (spectrum " $e$ " in Fig. 1) heated by the forward shock. The interstellar/circumstellar dust spectrum in Cas A has no associated gas line emission. The "broad" continuum (see Fig. $7 b$ of Ennis et al. 2006) is a combination of spectra c and e. Spectrum $\mathrm{c}$ has contamination from the shock-heated dust in projection, and for simplicity it is excluded in estimating the masses of the freshly formed dust (see $\S 5$ ). The featureless dust lacks the gentle peak around $26 \mu \mathrm{m}$ and also lacks the interstellar silicateemission feature between 9 and $11 \mu$ m observed in the spectra from the forward shock region. Most importantly, the featureless dust accompanies relatively strong $\mathrm{Si}$ and $\mathrm{S}$ ejecta lines and mostly from the interior of the remnant (blue region in Fig. $2 f$ ).

We generated a map of the $21 \mu \mathrm{m}$ peak dust by summing the emission over 19-23 $\mu \mathrm{m}$ after subtracting a baseline between 18-19 and 23-24 $\mu \mathrm{m}$. The line-free dust map (Fig. 2a) resembles the $[\mathrm{Ar} \mathrm{II}]$ and $[\mathrm{O} \mathrm{IV}]+[\mathrm{Fe}$ II $]$ ejecta-line maps, as shown in Figures $2 b$ and $2 c$, and we also find that the [Ne II] map is very similar to the [Ar II] map. The [Ar II] map shows a remarkable similarity to the $21 \mu \mathrm{m}$ peak dust map (Figs. $2 a$ and $2 b$ ), thereby 

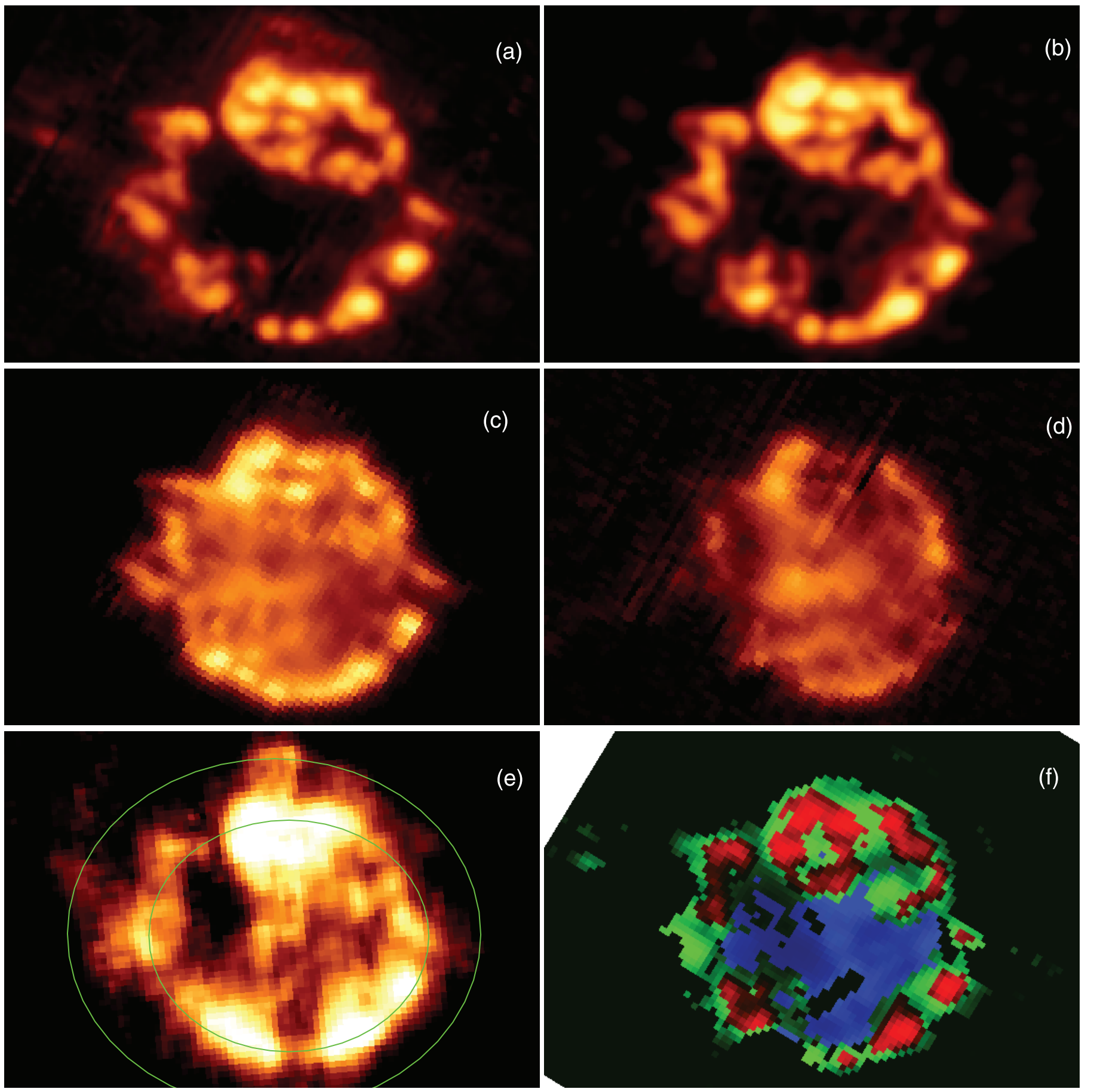

FIG. 2.-(a) $21 \mu \mathrm{m}$ dust map. A continuum map of 19-23 $\mu \mathrm{m}$ subtracted by the baselines of neighboring wavelengths. This dust map is remarkably similar to the (b) [Ar II] map (the resolutions are convolved to match each other). The image is centered at R.A. $23^{\mathrm{h}} 23^{\mathrm{m}} 25.86^{\mathrm{s}}$ and decl. $+58^{\circ} 49^{\prime} 14^{\prime \prime}(\mathrm{J} 2000.0)$ and covers an $7.87^{\prime} \times 5^{\prime}$ field of view. (c) A combination of [O Iv] and [ Fe II] line map at $26 \mu \mathrm{m}$. $(d)$ [Si II] $(34.8 \mu \mathrm{m})$ map. (e) MIPS $70 \mu \mathrm{m}$ map from Hines et al. (2004). The locations of the forward and reverse shock boundaries are marked as ellipses where we adjusted for elongation from Gotthelf et al. (2001) using long-exposure Chandra archival data (Hwang et al. 2004). ( $f$ ) Distributions of three major groups of dust types: $21 \mu \mathrm{m}$ peak dust regions are in red (spectrum a), weak $21 \mu \mathrm{m}$ dust regions are in green (spectrum b), and featureless dust regions are in blue (spectrum d in Fig. 1).

confirming that this dust is freshly formed in the ejecta. Maps of [Si II] (Fig. 2d) and [O IV]+[Fe II] (Fig. 2c) show significant emission at the center, revealing ejecta that have not yet been overrun by the reverse shock (unshocked ejecta). There is also [Si II] and $[\mathrm{O} \mathrm{IV}]+[\mathrm{Fe} \mathrm{II}]$ emission at the bright ring, indicating that some of the $\mathrm{Si}$ and $\mathrm{O}+\mathrm{Fe}$ ejecta have recently encountered the reverse shock. While the bright $\mathrm{O}+\mathrm{Fe}$ emission outlines the same bright ring structure as the $[\mathrm{Ar}$ II] and $21 \mu \mathrm{m}$ peak dust maps, the bright part of the Si shell shows a different morphology from the other ejecta maps.

We can characterize the spectra of our three dust classes by using the flux ratios between 17 and $21 \mu \mathrm{m}$ and between 21 and $24 \mu \mathrm{m}$. Although the spectra in Cas A show continuous changes in continuum shape from strong $21 \mu \mathrm{m}$ peak to weak $21 \mu \mathrm{m}$ peak and to featureless, we can locate regions where each of the three classes dominates. Figure $2 f$ shows the spatial distribution of our 
three dust classes where red, green, and blue indicate the $21 \mu \mathrm{m}$ peak dust, weak $21 \mu \mathrm{m}$ dust, and featureless dust, respectively. The flux ratios used to identify the three dust classes are as follows, where $I_{\lambda}$ is the flux density in the extracted spectrum at wavelength $\lambda(\mu \mathrm{m})$ :

1. $21 \mu \mathrm{m}$ peak dust. - We use the ratio $I_{21} / I_{24}>1+\sigma_{21 / 24}$, where $\sigma_{21 / 24}$ is the dispersion in $I_{21} / I_{24}$ over the remnant, which is equivalent to $I_{21} / I_{17} \geq 3.4$. The regions with $21 \mu \mathrm{m}$ peak dust coincide with the brightest ejecta.

2. Weak $21 \mu \mathrm{m}$ dust. - We use the ratio $1-\sigma_{21 / 24}<I_{21} / I_{24}<$ $1+\sigma_{21 / 24}$, which is equivalent to $2.3<I_{21} / I_{17}<3.4$. The regions showing the weak $21 \mu \mathrm{m}$ continuum shape mostly coincide with faint ejecta emission, but not always.

3. Featureless dust map.-We use the ratio $I_{21} / I_{24}<1-$ $\sigma_{21 / 24}$, which is equivalent to $I_{21} / I_{17}<2.3$. This ratio also picks out circumstellar dust heated by the forward shock, so we used several methods to exclude and mitigate contamination from circumstellar dust emission. First, using X-ray and radio maps, we excluded the forward shock regions at the edge of the radio plateau (Gotthelf et al. 2001). Second, there are highly structured "continuum-dominated" X-ray filaments across the face of the remnant that are similar to the exterior forward shock filaments and may be projected forward shock emission (DeLaney 2004). For our analysis, we excluded regions where there were infrared counterparts to the projected forward shock filaments. Third, for simplicity we excluded regions with gently rising spectra identified by curve $\mathrm{c}$ (the spectra that continues to rise to longer wavelengths) in Figure 1. This type of spectrum is mainly found on the eastern side of Cas A where there is an $\mathrm{H} \alpha$ region, the northeast jet, and other exterior optical ejecta (Fesen 2001), making it difficult to determine if the continuum emission is due to ejecta dust or circumstellar dust. However, note that some portion of the continuum in spectra $\mathrm{c}$ is freshly formed dust. We finally excluded regions where there was a noticeable correlation to optical quasi-stationary flocculi (van den Bergh 1971), which are dense circumstellar knots from the progenitor wind.

The featureless dust emission appears primarily across the center of the remnant, as shown in Figure $2 d$ (blue). The featureless dust is accompanied by relatively strong [Si II] and [S III] and $\left[\mathrm{O}_{\mathrm{IV}}+\mathrm{Fe}_{\mathrm{II}}\right]$ lines, as shown by spectrum d of Figure 1 . The $\left[\mathrm{O}_{\mathrm{IV}}\right]+$ [Fe II] line map (Fig. 2c) shows significant emission at the center as well as at the bright ring of the reverse shocked material. The [Si II] line map shows different morphology than other line maps and the $21 \mu \mathrm{m}$ peak dust map, depicting center-filled emission with a partial shell, as shown in Figure 2. This poses the following important question: why is the $\mathrm{Si}$ map more centerfilled than the Ar map? The answer is unclear because Si and Ar are both expected at similar depths in the nucleosynthetic layer (e.g., Woosley et al. 2002). The relatively faint infrared emission of $\mathrm{Si}$ and $\mathrm{S}$ at the reverse shock may imply relatively less $\mathrm{Si}$ and $\mathrm{S}$ in the reverse shock. We suspect it is because the $\mathrm{Si}$ and $\mathrm{S}$ have condensed to solid form such as $\mathrm{Mg}$ protosilicate, $\mathrm{MgSiO}_{3}$, $\mathrm{Mg}_{2} \mathrm{SiO}_{4}$, and $\mathrm{FeS}$. In contrast, Ar remains always in the gas and does not condense to dust, so it should be infrared or X-rayemitting gas. An alternate explanation is that the ionization in the interior is due to photoionization from the X-ray shell (see Hamilton \& Fesen 1988); in this case, the lack of interior Ar II relative to $\mathrm{Si}$ II might be due to its much higher ionization potential (16 eV compared to $8 \mathrm{eV}$ ). Theoretical models of nucleosynthesis, accounting for heating, photoionization, and column density of each element would be helpful for understanding the distribution of nucleosynthetic elements.
The Si and S emission detected at the interior is most likely unshocked ejecta where the revere shock has not yet overtaken the ejecta. The radial profile of unshocked ejecta is centrally peaked at the time of explosion, as shown by Chevalier \& Soker (1989). The radial profile of unshocked Fe ejecta is also expected to be center-filled for the $\sim 1000 \mathrm{yr}$ old Type Ia SNR of SN 1006 (Hamilton \& Fesen 1988). The morphology of the featureless dust resembles that of unshocked ejecta, supporting the conclusion that the featureless dust is also freshly formed dust. The spectrum in Figure 1 (curve d) shows the resolved two lines at 26 and at $35 \mu \mathrm{m}$. The two respective lines at $\sim 26 \mu \mathrm{m}$ may be resolved lines of [O IV] and [ $\mathrm{Fe}$ II], and at $\sim 35 \mu \mathrm{m}$ [Si II] and [ $\mathrm{Fe}$ II] (as expected that the unshocked ejecta near the explosion center have a low velocity); alternatively, they could be highly Dopplershifted lines (in this case the two lines at $26 \mu \mathrm{m}$ are both [O IV], and the two lines at $35 \mu \mathrm{m}$ are both [Si II]). The newly revealed unshocked ejecta deserves extensive studies; preliminary Dopplershifted maps were presented in DeLaney et al. (2006), and the detailed analysis of velocities and abundances of unshocked and shocked ejecta will be presented in future papers (T. DeLaney et al. 2008, in preparation; Smith et al. 2007).

\section{SPECTRAL FITTING AND DUST COMPOSITION}

We performed spectral fitting to the IRS continua using our example regions in Figure 1. Included in the fitting are MIPS 24 and $70 \mu \mathrm{m}$ fluxes (Hines et al. 2004), and the contribution of synchrotron emission (Figs. 3 and 5), estimated from the radio fluxes (DeLaney 2004) and Infrared Array Camera (IRAC) $3.6 \mu \mathrm{m}$ fluxes (Ennis et al. 2006). We measured synchrotron radiation components for each position using radio maps and assuming the spectral index $\alpha=-0.71$ (Rho et al. 2003), where $\log S \propto \alpha \log \nu$. Because the FWHM of $24 \mu \mathrm{m}$ is smaller than the IRS extracted region, the surface brightnesses for $24 \mu \mathrm{m}$ were measured using a $15^{\prime \prime}$ box, the same size as the area used for the extracted IRS LL spectra. We also made color corrections to each MIPS $24 \mu \mathrm{m}$ data point based on each IRS spectrum and band-filter shape; the correction was as high as $25 \%$ for some positions. While the uncertainty of calibration errors in IRAC is $3 \%-4 \%$, that of MIPS $24 \mu \mathrm{m}$ is better than $10 \%$. The MIPS $70 \mu \mathrm{m}$ image (Hines et al. 2004), shown in Figure $2 e$, clearly resolves Cas A from background emission, unlike the $160 \mu \mathrm{m}$ image (Krause et al. 2004). Most of the bright $70 \mu \mathrm{m}$ emission appears at the bright ring and corresponds to the $21 \mu \mathrm{m}$ dust map and the shocked ejecta, particularly [Ar II], indicating that the $70 \mu \mathrm{m}$ emission is primarily from freshly formed dust in the ejecta. The $70 \mu \mathrm{m}$ emission also appears at the interior, as shown in Figure $2 e$. We measured the brightness for $70 \mu \mathrm{m}$ within a circle of radius $20^{\prime \prime}$ for each position, accounting for the pointspread function (note that when the emission is uniform, the aperture size does not affect the surface brightness). We estimated the uncertainties of the $70 \mu \mathrm{m}$ fluxes to be as large as $30 \%$. The largest uncertainty comes from background variation due to cirrus structures based on our selection of two background areas, $5^{\prime}$ to the northwest and south of the Cas A.

The dust continuum is fit with the Planck function $\left[B_{\nu}(T)\right]$ multiplied by the absorption efficiency $\left(Q_{\text {abs }}\right)$ for various dust compositions, varying the amplitude and temperature of each component. To determine the dust composition, we consider not only the grain species predicted by the model of dust formation in $\mathrm{SNe}$ (TF01, N03), but also Mg protosilicates (ADM99) and $\mathrm{FeO}$ (Henning et al. 1995) as possible contributors to the $21 \mu \mathrm{m}$ feature. The optical constants of the grain species used in the calculation are the same as those of Hirashita et al. (2005) except for amorphous Si (Piller 1985), amorphous $\mathrm{SiO}_{2}$ (Philipp 1985), 


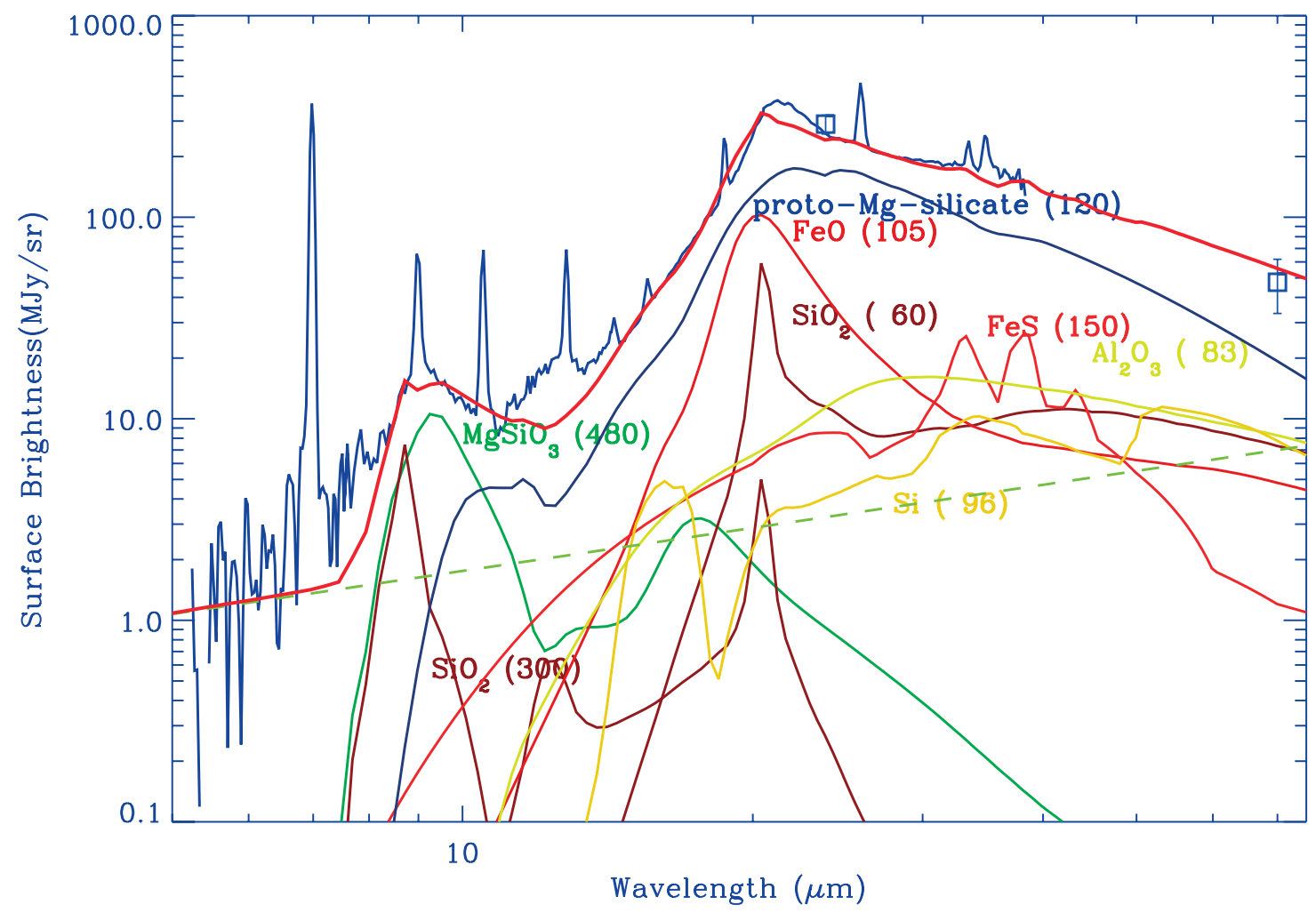

FIG. 3. - $21 \mu \mathrm{m}$ peak dust spectrum superposed on the dust fit of model A. A Spitzer IRS spectrum toward a bright part of the northern shell fitted with dust compositions of $\mathrm{Mg}$ protosilicate, $\mathrm{MgSiO}_{3}, \mathrm{SiO}_{2}, \mathrm{FeO}$, and $\mathrm{Al}_{2} \mathrm{O}_{3}$. The compositions suggest that the dust forms around inner oxygen and $\mathrm{S}$-Si layers. The data and the total fit are shown in blue and thick red lines, respectively, and MIPS fluxes are marked with squares. The dust temperatures are shown in parentheses, and the dotted lines are from the second temperature components. Synchrotron continuum contribution (green dashed line) is estimated based on the radio fluxes and IRAC $3.6 \mu \mathrm{m}$ image.

amorphous $\mathrm{Al}_{2} \mathrm{O}_{3}$ (Begemann et al. 1997), and $\mathrm{FeO}$ (Henning et al. 1995); we apply Mie theory (Bohren \& Huffman 1983) to calculate the absorption efficiencies, $Q_{\mathrm{abs}}$, assuming the grains are spheres of radii $a=0.01 \mu \mathrm{m}$. We fit both amorphous and crystalline grains for each composition, but it turned out that the fit results in Cas A (see $\S 3$ ) favor amorphous over crystalline grains. Thus, default grain composition hereafter indicates amorphous. For $\mathrm{Mg}$ protosilicate, the absorption coefficients are evaluated from the mass absorption coefficients tabulated in Dorschner et al. (1980), and we assume that the absorption coefficient varies as $\lambda^{-2}$ for $\lambda>40 \mu \mathrm{m}$, typical for silicates. We fit the flux density for each spectral type using scale factors $C_{i}$ for each grain type $i$, such that $F_{\nu}^{i}=\Sigma_{i} C_{i} B_{\nu} Q_{\mathrm{abs}, i} / a$. Note that the calculated values of $Q_{\mathrm{abs}} / a$ are independent of the grain size as long as $2 \pi|m| a / \lambda \ll 1$, where $m$ is the complex refractive index. Thus, the derived scale factor $C_{i}$ as well as the estimated dust mass (see
$\S 4)$ are independent of the radius of the dust. The dust compositions of the best fits are summarized in Table 1 .

The strong $21 \mu \mathrm{m}$ peak dust is best fit by $\mathrm{Mg}$ protosilicate, amorphous $\mathrm{SiO}_{2}$, and $\mathrm{FeO}$ grains (with temperatures of $60-$ $120 \mathrm{~K}$ ), as shown in Figure 3. These provide a good match to the $21 \mu \mathrm{m}$ feature. ADM99 suggested that the $21 \mu \mathrm{m}$ feature is best fit by $\mathrm{Mg}$ protosilicate, while $\mathrm{D} 01$ suggested it is best fit by $\mathrm{SiO}_{2}$ instead. We found, however, that $\mathrm{SiO}_{2}$ produced a $21 \mu \mathrm{m}$ feature that was too sharp. We also fit the observations using $\mathrm{Mg}_{2} \mathrm{SiO}_{4}$, which exhibits a feature around $20 \mu \mathrm{m}$ and the overall variation of absorption coefficients of $\mathrm{Mg}_{2} \mathrm{SiO}_{4}$ whose wavelength might be similar, to that of $\mathrm{Mg}$ protosilicate (Dorschner et al. 1980; Jäger et al. 2003). However, with $\mathrm{Mg}_{2} \mathrm{SiO}_{4}$, the fit is not as good as that of $\mathrm{Mg}$ protosilicate, not only at the $21 \mu \mathrm{m}$ peak, but also at shorter $(10-20 \mu \mathrm{m})$ and longer $(70 \mu \mathrm{m})$ wavelengths. Thus, we use $\mathrm{Mg}$ protosilicate and $\mathrm{SiO}_{2}$ as silicates to fit the $21 \mu \mathrm{m}$ peak

TABLE 1

Properties of Freshly Formed Dust in Cas A

\begin{tabular}{|c|c|c|c|c|c|}
\hline $21 \mu \mathrm{m}$ peak (spectrum a) ........... & A & Mg protosilicate, $\mathrm{MgSiO}_{3}, \mathrm{SiO}_{2}, \mathrm{FeO}, \mathrm{FeS}, \mathrm{Si}, \mathrm{Al}_{2} \mathrm{O}_{3}$ & $\mathrm{Ar}$ & Inner $\mathrm{O}, \mathrm{S}-\mathrm{Si}$ & 0.0030 \\
\hline Weak $21 \mu \mathrm{m}$ (spectrum b).......... & $\mathrm{C}$ & $\mathrm{C}$ glass, $\mathbf{F e O}, \mathrm{Al}_{2} \mathrm{O}_{3}, \mathrm{Si}, \mathrm{Mg}_{2} \mathrm{SiO}_{4}$ & $\mathrm{Ne}, \mathrm{Si}, \mathrm{Ar}(\mathrm{S}, \mathrm{O}+\mathrm{Fe})$ & C burning & 0.0180 \\
\hline Weak $21 \mu \mathrm{m}$ & $\mathrm{D}$ & $\mathrm{C}$ glass, $\mathrm{FeO}, \mathrm{Al}_{2} \mathrm{O}_{3}, \mathrm{Si}, \mathrm{FeS}$, Mg protosilicate & $\mathrm{Ne}, \mathrm{Si}, \mathrm{Ar}(\mathrm{S}, \mathrm{O}+\mathrm{Fe})$ & C burning & 0.0157 \\
\hline Featureless (spectrum d) ............. & $\mathrm{E}$ & $\mathrm{MgSiO}_{3}, \mathbf{S i}, \mathrm{FeS}, \mathrm{Fe}, \mathrm{Mg}_{2} \mathrm{SiO}_{4}$ & $\mathrm{Si}, \mathrm{S},(\mathrm{O}+\mathrm{Fe})$ & $\mathrm{O}, \mathrm{Al}$ burning $(\mathrm{Fe}-\mathrm{Si}-\mathrm{S})$ & 0.0245 \\
\hline
\end{tabular}

\footnotetext{
${ }^{\mathrm{a}}$ Compositions in the best fit, where a few primary compositions are set in bold, and alternative dust compositions are set in italics.
} 


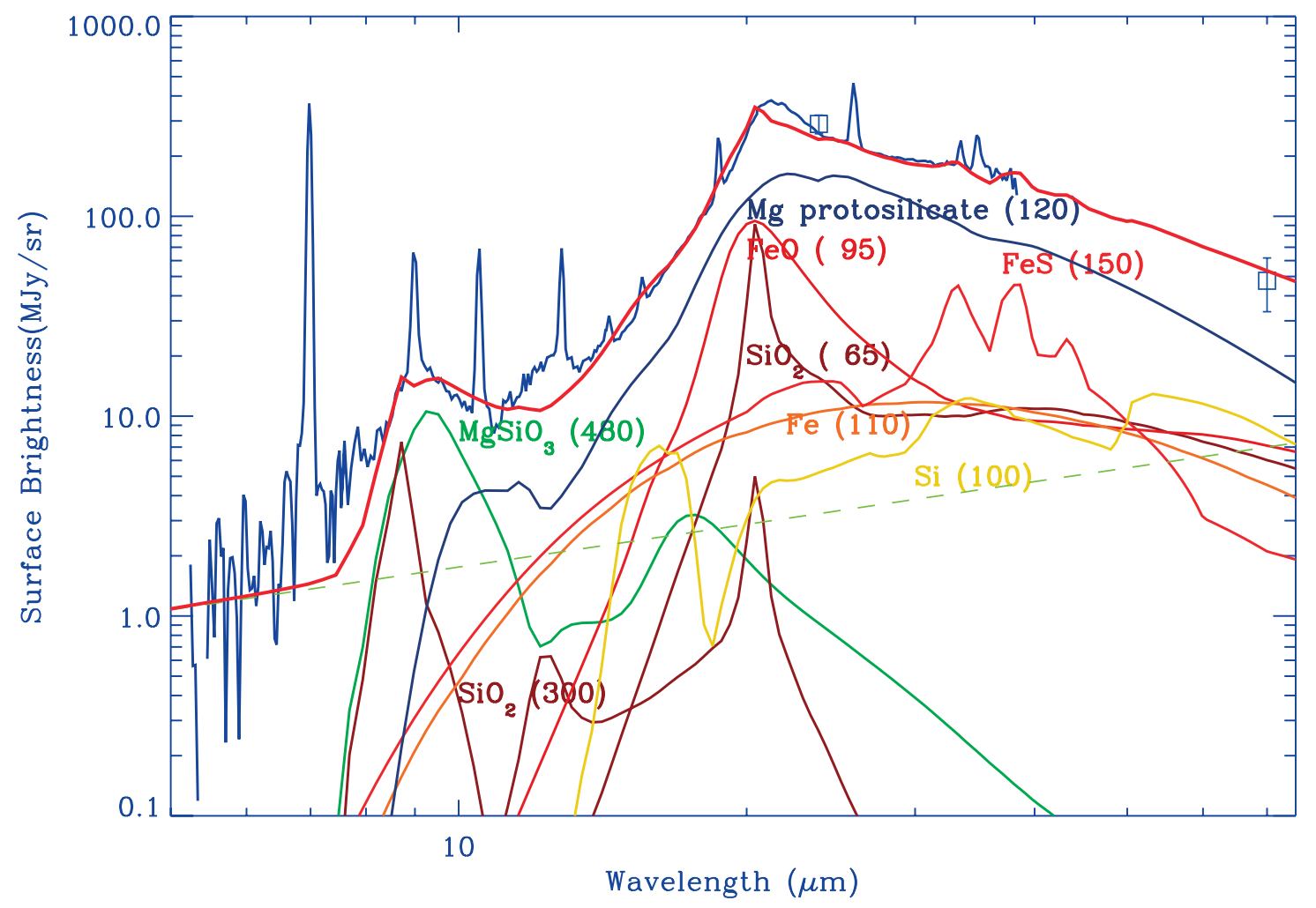

FIG. 4. $-21 \mu \mathrm{m}$ peak dust superposed on the dust fit of model B.

dust feature. The fit with $\mathrm{Mg}$ protosilicate, $\mathrm{SiO}_{2}$, and $\mathrm{FeO}$ is improved by adding aluminum oxide $\left(\mathrm{Al}_{2} \mathrm{O}_{3}, 83 \mathrm{~K}\right)$ and $\mathrm{FeS}$ $(150 \mathrm{~K})$, where $\mathrm{Al}_{2} \mathrm{O}_{3}$ improved the overall continuum shape between 10 and $70 \mu \mathrm{m}$ and FeS improved the continuum between 30 and $40 \mu \mathrm{m}$ (underneath the lines of $\mathrm{Si}, \mathrm{S}$, and $\mathrm{Fe}$ ), as shown in Figure 3. The silicate composition is responsible for the $21 \mu \mathrm{m}$ peak, suggesting that the dust forms around the inneroxygen and S-Si layers and is consistent with Ar being one of the oxygen-burning products. We also include amorphous $\mathrm{MgSiO}_{3}$ $(480 \mathrm{~K})$ and $\mathrm{SiO}_{2}(300 \mathrm{~K})$ to account for the emission feature around $9.8 \mu \mathrm{m}$. The composition of the low-temperature (40$90 \mathrm{~K}$ ) dust component necessary for reproducing $70 \mu \mathrm{m}$ is rather unclear. Either $\mathrm{Al}_{2} \mathrm{O}_{3}(80 \mathrm{~K})$ (model A in Table 1) or Fe $(110 \mathrm{~K}$ ) (model B in Table 1 and Fig. 4) can fit equally well, as listed in Table 1 . We could use carbon instead of $\mathrm{Al}_{2} \mathrm{O}_{3}$ or $\mathrm{Fe}$, but the line and dust compositions suggest that the emission is from inner $\mathrm{O}$, $\mathrm{S}-\mathrm{Si}$ layers, where carbon dust is not expected. There are still residuals in the fit from the feature peaking at $21 \mu \mathrm{m}(20-23 \mu \mathrm{m})$ and an unknown dust feature at $11-12.5 \mu \mathrm{m}$ (it is not a part of typical PAH feature), as shown in Figure 3. The former may be due to nonspherical grains or different sizes of grains. The mass and temperature of each composition are listed in detail in Table 2.

The weak $21 \mu \mathrm{m}$ continuum is fit by $\mathrm{FeO}$ and $\mathrm{Mg}_{2} \mathrm{SiO}_{4}$ or $\mathrm{Mg}$ protosilicate (models $\mathrm{C}$ and $\mathrm{D}$ in Table 1) since the curvature of the continuum changes at 20-21 $\mathrm{m}$, as shown in Figure 5. To fit the rest of the spectrum, we use glassy carbon dust and $\mathrm{Al}_{2} \mathrm{O}_{3}$ grains. The glassy carbon grains $(220 \mathrm{~K})$ can account for the smooth curvature in the continuum between 8 and $14 \mu \mathrm{m}$. Carbon dust $(80 \mathrm{~K})$ and $\mathrm{Al}_{2} \mathrm{O}_{3}(100 \mathrm{~K})$ contribute to the continuum between 15 and $25 \mu \mathrm{m}$. We could use Fe dust instead, but we suspect carbon dust because of the presence of relatively strong $\mathrm{Ne}$ line emission with the weak $21 \mu \mathrm{m}$ dust class. $\mathrm{Ne}, \mathrm{Mg}$, and $\mathrm{Al}$ are all carbon-burning products. We cannot fit the spectrum replacing carbon by $\mathrm{Al}_{2} \mathrm{O}_{3}$ with a single or two temperatures because $Q_{\text {abs }} / a$ of $\mathrm{Al}_{2} \mathrm{O}_{3}$ has a shallow bump around $27 \mu \mathrm{m}$; thus, the fit requires three-temperature components of $\mathrm{Al}_{3} \mathrm{O}_{2}$ or a combination of twotemperature components of $\mathrm{Al}_{3} \mathrm{O}_{2}$ and a temperature component of carbon. The continuum between 33 and $40 \mu \mathrm{m}$ (underneath the lines of $\mathrm{Si}, \mathrm{S}$, and $\mathrm{Fe}$ ) can be optimally fit by $\mathrm{FeS}$ grains.

The $70 \mu \mathrm{m}$ image shown in Figure $2 e$ shows interior emission similar to the unshocked ejecta, but that may also be due to projected circumstellar dust at the forward shock. In order to fit the featureless spectrum out to $70 \mu \mathrm{m}$, we must first correct for possible projected circumstellar dust emission. The exterior forward shock emission is most evident in the northern and northwestern shell. Taking the typical brightness in the northwest shell $\left(\sim 20 \mathrm{MJy} \mathrm{sr}^{-1}\right)$, and assuming that the forward shock is a shell with $12 \%$ radial thickness, the projected brightness is less than $4 \%-10 \%$ of the interior emission ( $40 \mathrm{MJy} \mathrm{sr}^{-1}$ after background subtraction). We assume that the remaining wide-spread interior $70 \mu \mathrm{m}$ emission is from relatively cold, unshocked ejecta. Using the "corrected" $70 \mu \mathrm{m}$ flux, the featureless spectra are equally reproduced by three models (models E, F, and G) in Table 1 and Figures 6 and 7. All fits include $\mathrm{MgSiO}_{3}, \mathrm{FeO}$, and $\mathrm{Si}$, and either aluminum oxide, $\mathrm{Fe}$, or a combination of the two are required at long wavelength. Carbon dust can also produce featureless spectra at low temperature, but we exclude this composition because of the lack of $\mathrm{Ne}$ (produced from carbon burning). Aluminum oxide and Fe dust are far more likely to be associated with the unshocked ejecta because they result from O-burning and Si-burning, respectively, and the unshocked ejecta exhibit $\mathrm{Si}, \mathrm{S}$, and $\mathrm{O}+\mathrm{Fe}$ line emission. However, one of the key challenges in SN ejecta dust is to understand featureless dust such as $\mathrm{Fe}, \mathrm{C}$, and aluminum oxide, and to link it to the associated nucleosynthetic products.

\section{DUST MASS}

We estimated the amount of freshly formed dust in Cas A based on our dust model fit to each of the representative $21 \mu \mathrm{m}$ 


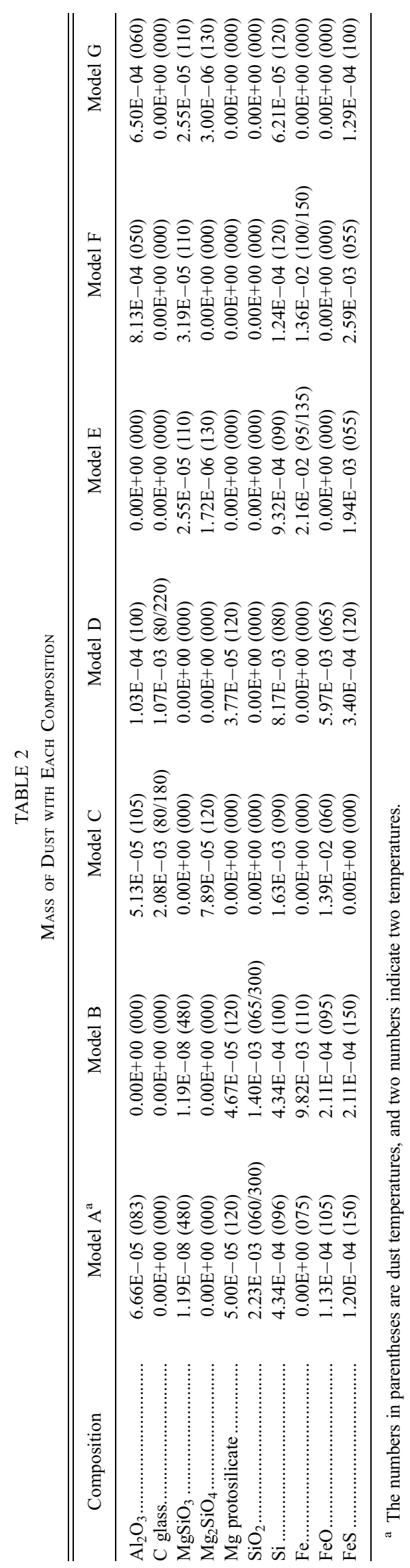




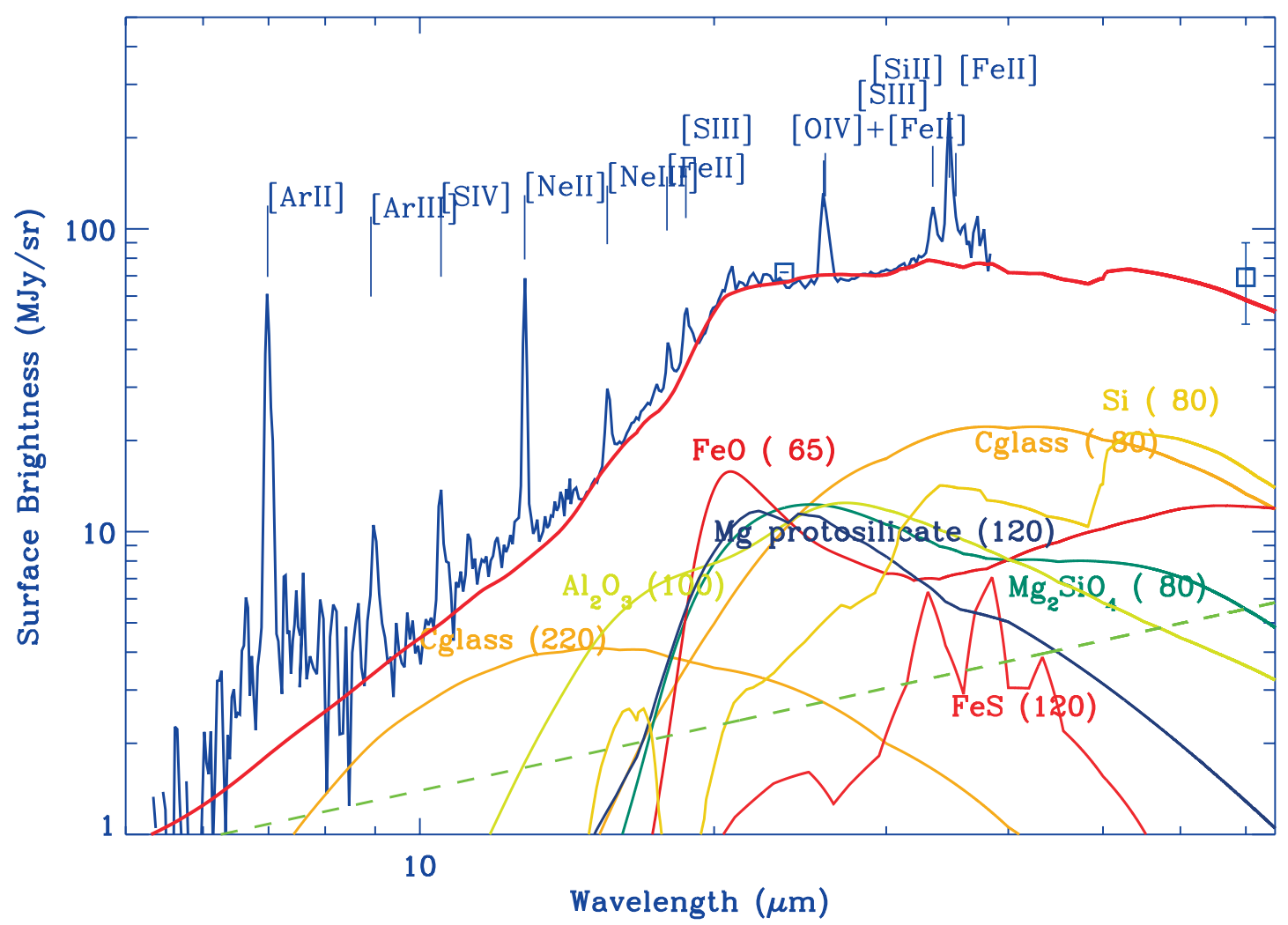

Fig. 5.-Weak $21 \mu \mathrm{m}$ dust superposed on the dust fit of model D. A second type of dust continuum in Cas A. The distribution of this type of dust is shown in Fig. $2 f$ in green.

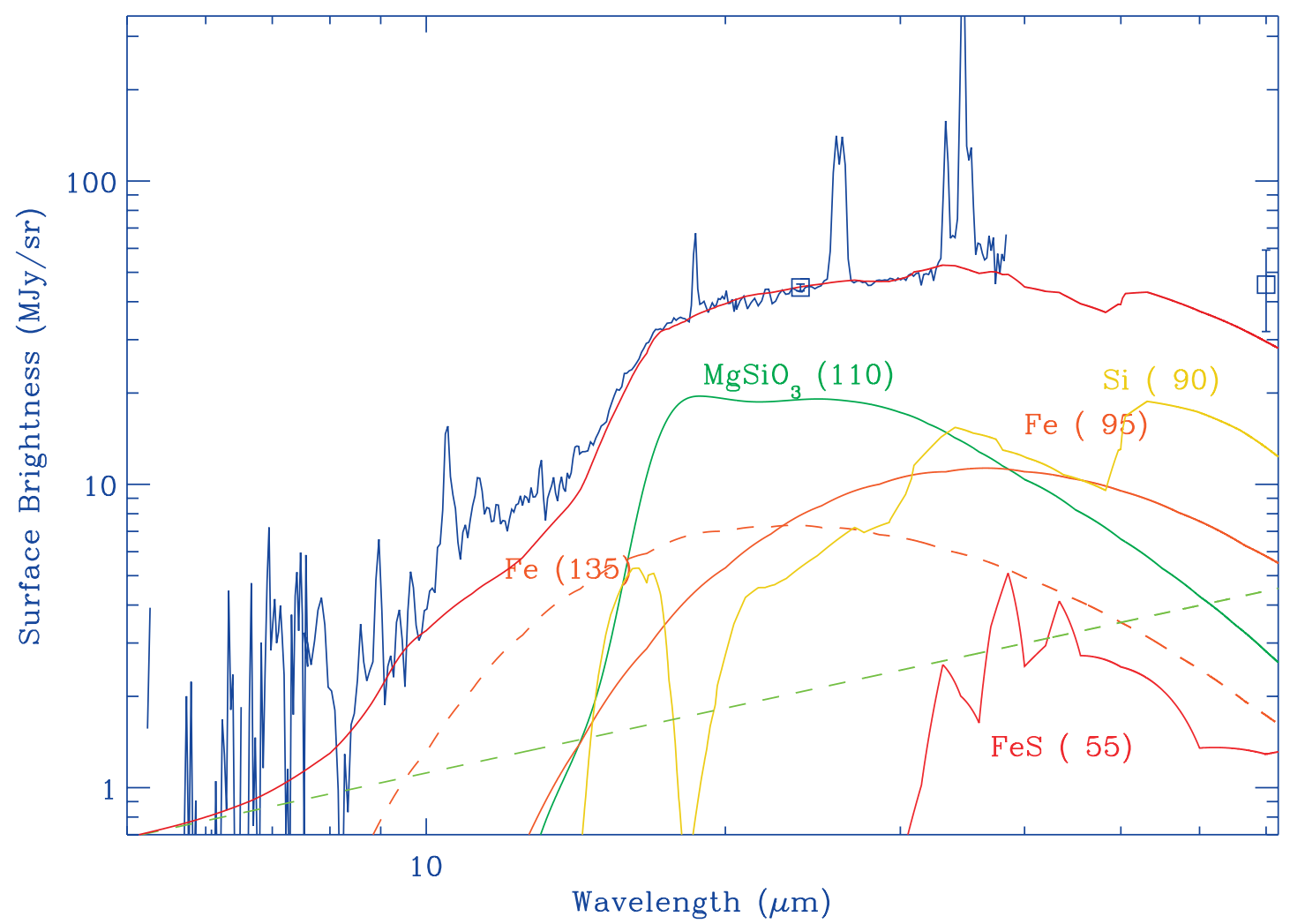

Fig. 6.-Featureless dust spectrum. The continuum can be fit with $\mathrm{MgSiO}_{3}$ and $\mathrm{Fe}$ (model E). The featureless spectra is accompanied with $\mathrm{S}$, $\mathrm{Si}$, and $\mathrm{O} / \mathrm{Fe}$ lines. The green dashed line is the predicted synchrotron emission model. 


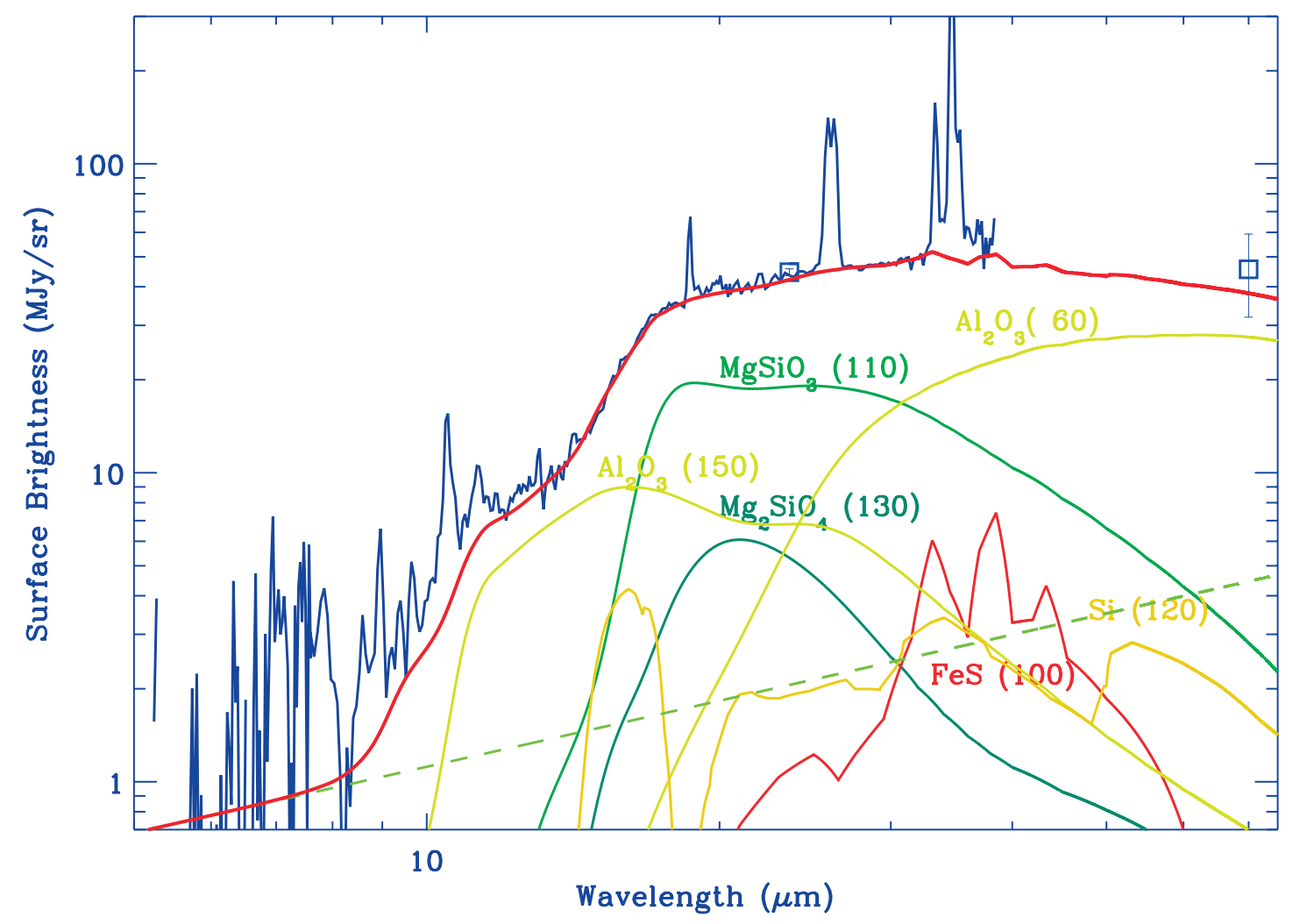

FIG. 7.-Featureless dust spectrum. The continuum can be fit with $\mathrm{Al}_{2} \mathrm{O}_{3}$ and $\mathrm{MgSiO}_{3}$ (model G).

peak, weak $21 \mu \mathrm{m}$, and featureless spectra (Fig. 1). The dust mass of $i$-grain type is given by

$$
M_{\mathrm{dust}, i}=\frac{F_{\nu}^{i} d^{2}}{B_{\nu}\left(T_{d, i}\right) \kappa_{i}}=\frac{F_{\nu}^{i} d^{2}}{B_{\nu}\left(T_{d, i}\right)} \frac{4 \rho_{i} a}{3 Q_{\mathrm{abs}, i}},
$$

where $F_{\nu}^{i}$ is the flux from $i$-grain species, $d$ is the distance, $B_{\nu}$ is the Planck function, $\rho_{i}$ is the bulk density, and $a$ is the dust particle size. By employing the scale factor $C_{i}$ and the dust temperature $T_{d, i}$ derived from the spectral fit, the total dust mass is given by $M_{\text {dust }}=\sum_{i} \rho_{i} \Omega d^{2} C_{i} / 3$, where $\Omega$ is the solid angle of the source. The total mass of the $21 \mu \mathrm{m}$ peak dust is then determined by summing the flux of all the pixels in the $21 \mu \mathrm{m}$ peak dust region (red region in Fig. $2 f$ ) and assuming each pixel in this region has the same dust composition as the spectrum in Figure 3. We took the same steps for the weak $21 \mu \mathrm{m}$ dust and the featureless dust.

The estimated total masses for each type of dust using a distance of $3.4 \mathrm{kpc}$ (Reed et al. 1995) are listed in Table 1. Using the least massive composition in Table 1 for each of the three dust classes yields a total mass of $0.020 M_{\odot}$ (the sum of masses from models A, D, and F). Using the most massive composition for each of the three dust classes yields a total mass of $0.054 M_{\odot}$ (the sum of masses from models B, C, and E). The primary uncertainty in the total dust mass between 0.020 and $0.054 M_{\odot}$ is due to the selection of the dust composition, in particular for the featureless dust.

We also extracted a global spectrum of Cas A, but excluding most of the exterior forward shock regions. The spectrum is well fit with the combination of our three types of dust (including all compositions from models A to G), as shown in Figure 8. We used the dust composition of models $\mathrm{A}-\mathrm{G}$ as a guideline in fitting the global spectrum, because the dust features (which were noticeable in representative spectra) were smeared out. Our goal in fitting the global spectrum is to confirm consistency between the mass derived from global spectrum and that derived from representative spectra described above. The total estimated mass from the global spectrum fit is $\sim 0.028 M_{\odot}$, being consistent with the mass determined from the individual fits to each dust class. The respective dust mass for each grain composition is listed in Table 3. The masses of $\mathrm{MgSiO}_{3}, \mathrm{SiO}_{2}, \mathrm{FeS}$, and $\mathrm{Si}$ are more than a factor of 10-100 smaller than the predictions; the predictions (N03 and TF01) also have the dust features at $9 \mu \mathrm{m}$ for $\mathrm{MgSiO}_{3}, 21 \mu \mathrm{m}$ for $\mathrm{SiO}_{2}$, and $30-40 \mu \mathrm{m}$ for $\mathrm{FeS}$ stronger than the observed spectra if the dust mass is increased. The carbon mass is also a factor of 10 lower than the predictions. We were not able to fit the data with as much carbon dust mass as expected, even if we use the maximum carbon contribution allowed from the spectral fits.

\section{DISCUSSION}

We find that an estimated total freshly formed dust mass of $0.020-0.054 M_{\odot}$ is required to produce the mid-infrared continuum up to $70 \mu \mathrm{m}$. The dust mass we derive is orders of magnitude higher than the two previous infrared estimates of $3.5 \times$ $10^{-3}$ and $7.7 \times 10^{-5} M_{\odot}$, which are derived by extrapolation from $1.6 \times 10^{-4} M_{\odot}(\mathrm{D} 01)$ and $2.8 \times 10^{-6} M_{\odot}$ (ADM99) for selected knots, respectively. One of the primary reasons for our higher mass estimate is that we include fluxes up to $70 \mu \mathrm{m}$, while the fits in D01 and ADM99 accounted for dust emission only up to 30 and $\sim 40 \mu \mathrm{m}$, respectively. The cold dust (40-150 K) has much more mass than the warmer $(>150 \mathrm{~K})$ dust. In addition, our IRS mapping over nearly the entire extent of Cas A with higher spatial and spectral resolutions provides more accurate measurements, while D01 and ADM99 covered only a portion of the remnant. In addition, ADM99 use only Mg protosilicate dust; the 


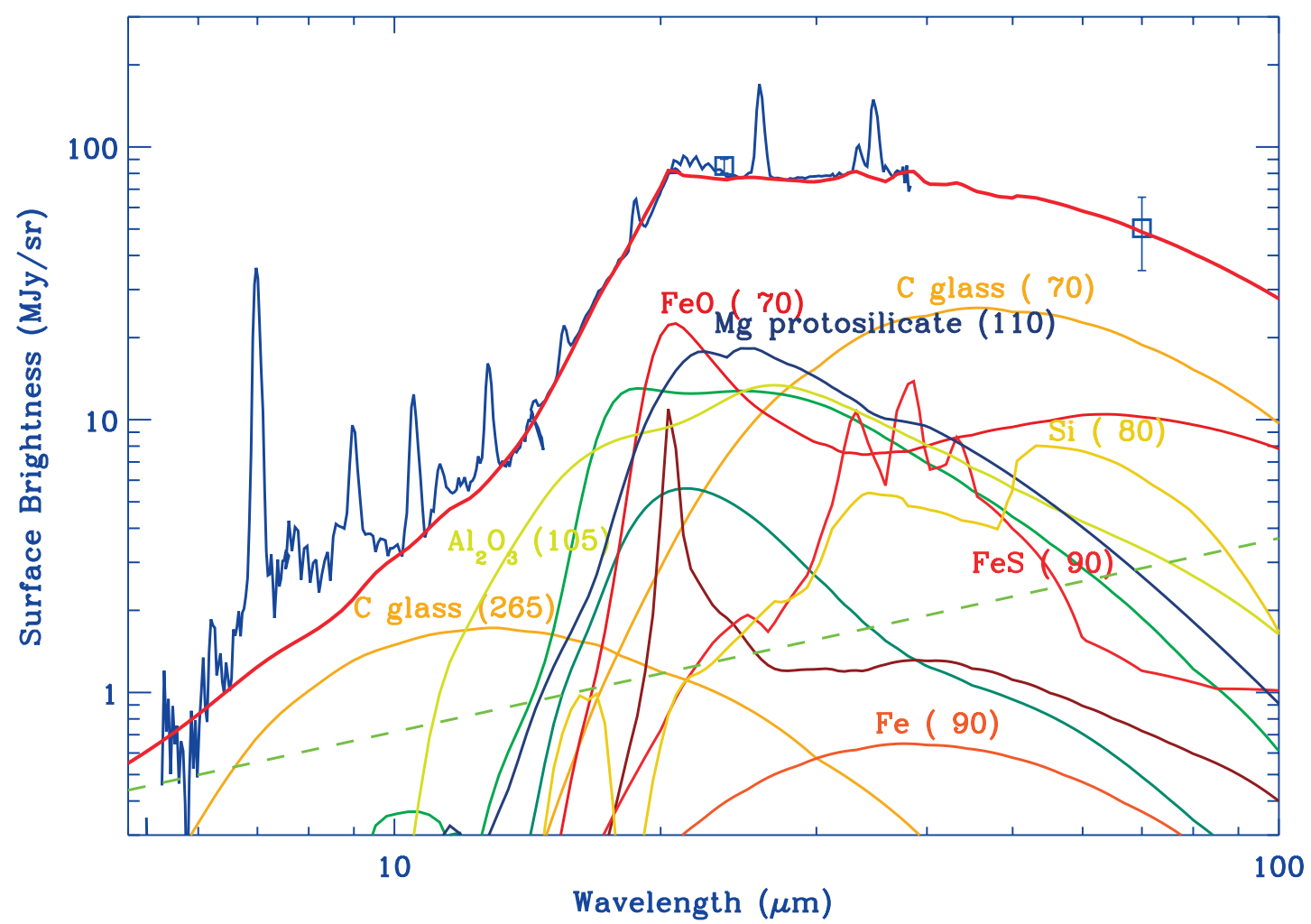

Fig. 8. - Total spectrum of Cas A and the dust fit of grains with a few primary contributions of grain models. The dark green and brown lines are $\mathrm{MgSiO}_{3}(100 \mathrm{~K})$ and $\mathrm{SiO}_{2}(65 \mathrm{~K})$, respectively. The green dashed line is the predicted synchrotron emission model.

absorption coefficient for $\mathrm{Mg}$ protosilicate is a few times larger than those of other compositions.

Our dust mass estimate is also at least 1 order of magnitude higher than the estimate of $3 \times 10^{-3} M_{\odot}$ by Hines et al. (2004). They fitted MSX and Spitzer MIPS data with Mg protosilicate. Note that they used only one composition. They derived a freshly synthesized dust mass of $3 \times 10^{-3} M_{\odot}$ at a temperature of $79-$ $82 \mathrm{~K}$ and a smaller dust mass of $5 \times 10^{-6} M_{\odot}$ at a higher temperature of $226-268 \mathrm{~K}$, and they explained that the mass estimate depends on the chosen dust temperature. As ADM99 mentioned, the absorption coefficient for $\mathrm{Mg}$ protosilicate is a few times larger than those of other compositions. Therefore, even including the long-wavelength data, the estimated mass was small since only $\mathrm{Mg}$ protosilicate was modeled. With the photometry in Hines et al. (2004), one could easily fit the data with only Mg protosilicate and would not need additional grain compositions. However, with the accurate IRS data, many dust features and the detailed continuum shape could not be fit solely with the $\mathrm{Mg}$ protosilicate. Note that the continuum shapes of weak $21 \mu \mathrm{m}$ dust and featureless dust are very different from the shape of the protosilicate absorption coefficient. Therefore, it was necessary to include many other compositions in order to reproduce the observed IRS spectra.

It should be noted here that, in contrast with the previous works, we introduced $\mathrm{Si}$ - and Fe-bearing materials such as $\mathrm{Si}$, $\mathrm{Fe}, \mathrm{FeS}$, and $\mathrm{FeO}$. We explain why we included such dust in our model fitting as follows. First, we included Si and Fe dust because these elements are significant outputs of nucleosynthesis;

TABLE 3

Mass of Dust with Each Composition

\begin{tabular}{|c|c|c|c|c|}
\hline Composition & Predicted $\left(M_{\odot}\right)$ & Model $(\mathrm{A}+\mathrm{D}+\mathrm{G})^{\mathrm{a}}$ & Model $(\mathrm{B}+\mathrm{C}+\mathrm{E})^{\mathrm{b}}$ & Mass $\left(\right.$ global) ${ }^{\mathrm{c}}$ \\
\hline $\mathrm{Al}_{2} \mathrm{O}_{3} \ldots \ldots \ldots \ldots \ldots$ & $2.40 \mathrm{E}-04 \sim 9.00 \mathrm{E}-03$ & $8.20 \mathrm{E}-04$ & $5.1300 \mathrm{E}-05$ & $1.22 \mathrm{E}-04(105)$ \\
\hline 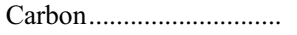 & $7.00 \mathrm{E}-02 \sim 3.00 \mathrm{E}-01$ & $1.07 \mathrm{E}-03$ & $2.0767 \mathrm{E}-03$ & $2.04 \mathrm{E}-03(070 / 265)$ \\
\hline $\mathrm{MgSiO}_{3}$ & $2.00 \mathrm{E}-03 \sim 7.00 \mathrm{E}-3$ & $2.55 \mathrm{E}-05$ & $2.5500 \mathrm{E}-05$ & $1.65 \mathrm{E}-04(110)$ \\
\hline $\mathrm{Mg}_{2} \mathrm{SiO}_{4} \ldots \ldots \ldots \ldots \ldots$ & $3.70 \mathrm{E}-02 \sim 4.40 \mathrm{E}-1$ & $3.00 \mathrm{E}-06$ & $8.0620 \mathrm{E}-05$ & $3.21 \mathrm{E}-05(120)$ \\
\hline $\mathrm{Mg}$ protosilicate ............... & None $^{\mathrm{d}}$ & $8.77 \mathrm{E}-05$ & $4.6710 \mathrm{E}-05$ & $6.70 \mathrm{E}-05(110)$ \\
\hline $\mathrm{SiO}_{2} \ldots \ldots \ldots \ldots \ldots \ldots \ldots$ & $2.50 \mathrm{E}-02 \sim 1.400 \mathrm{E}-01$ & $2.23 \mathrm{E}-03$ & $1.3964 \mathrm{E}-03$ & $1.35 \mathrm{E}-03(065)$ \\
\hline $\mathrm{Si}$ & $7.00 \mathrm{E}-02 \sim 3.00 \mathrm{E}-01$ & $8.66 \mathrm{E}-03$ & $2.9989 \mathrm{E}-03$ & 4.42E-03 (080) \\
\hline Fe & $2.00 \mathrm{E}-02 \sim 4.00 \mathrm{E}-02$ & $0.00 \mathrm{E}+00$ & $3.1459 \mathrm{E}-02$ & $1.03 \mathrm{E}-02(090)$ \\
\hline $\mathrm{FeO}$ & None ${ }^{\mathrm{d}}$ & $6.08 \mathrm{E}-03$ & $1.4136 \mathrm{E}-02$ & $6.23 \mathrm{E}-03(070)$ \\
\hline $\mathrm{FeS}$ & $4.00 \mathrm{E}-02 \sim 1.10 \mathrm{E}-01$ & $5.90 \mathrm{E}-04$ & $2.1501 \mathrm{E}-03$ & $2.90 \mathrm{E}-03(090)$ \\
\hline
\end{tabular}

a The sum of the masses using the least massive composition among models.

b The sum of the masses using the most massive compositions.

c The mass using the global spectrum.

${ }^{\mathrm{d}}$ See the text for details. 
indeed, Woosley \& Weaver (1995) show that Si and Fe are primary products in the innermost layers of the ejecta. Second, we observed strong $\mathrm{Si}$ and $\mathrm{Fe}$ lines in the infrared and X-ray spectra; strong Si lines were detected in the Spitzer spectra, as shown in Figure 1 (also see D01), and the Fe line detection at $17.9 \mu \mathrm{m}$ is also shown in Figure 5. (The Fe maps at 17.9 and $1.64 \mu \mathrm{m}$ were presented in Ennis et al. [2006] and Rho et al. [2003], respectively.) $\mathrm{Si}$ and $\mathrm{Fe}$ lines from ejecta are also bright in X-ray emission (Hwang et al. 2000). Third, dust such as $\mathrm{Si}, \mathrm{Fe}, \mathrm{FeO}$, and $\mathrm{FeS}$ is predicted to form in the ejecta of Population III supernovae (N03). TF01 and $\mathrm{N} 03$ predict $\mathrm{Fe}_{3} \mathrm{O}_{4}$ instead of $\mathrm{FeO}$ in the uniformly mixed ejecta where the elemental composition is oxygen-rich, but the kind of iron-bearing grains in oxygen-rich layers of the ejecta is still uncertain, partly because the surface energy of iron is very sensitive to the concentration of impurities such as $\mathrm{O}$ and S (as was discussed by Kozasa \& Hasegawa 1988) and partly because the chemical reactions at the condensation of Fe-bearing dust is not well understood. Depending on the elemental composition and the physical conditions in the ejecta, it is possible that $\mathrm{Fe}, \mathrm{FeO}$, and/or $\mathrm{FeS}$ form in the oxygen-rich layers of Galactic SNe. The observations of Cas A favor $\mathrm{FeO}$ dust over $\mathrm{Fe}_{3} \mathrm{O}_{4}$, in order to match the spectral shape of the $21 \mu \mathrm{m}$ peak dust and the weak $21 \mu \mathrm{m}$ dust. This aspect should be explored theoretically in comparison with the observations in the future.

Our total mass estimate is also about 1 order of magnitude higher than the estimate of $6.9 \times 10^{-3} M_{\odot}$ by Dwek et al. (1987), who used IRAS fluxes (possibly confused by background cirrus) and assumed a silicate-type dust as stellar or supernova condensates being present in supernova cavity and heated up by the reverse shock. Our estimated mass is much less than $1 M_{\odot}$, which Wilson \& Batrla (2005) suggested may still be associated with the ejecta, after accounting for results of high-resolution $\mathrm{CO}$ observations. Our estimated mass of $0.020-0.054 M_{\odot}$ is only derived for wavelengths up to $70 \mu \mathrm{m}$, so it is still possible that the total freshly formed dust mass in Cas A is higher than our estimate because there may be colder dust present. Future longer wavelength observations with Herschel, SCUBA-2, and ALMA are required to determine if this is the case. Also note that we did not include any mass from fast-moving knots projected into the same positions as the forward shock, such as in the northeast and southwest jets, and the eastern portions of the SNR outside the $21 \mu \mathrm{m}$ peak dust region (see Fig. $2 e$ ), because such dust could not be cleanly separated from the interstellar/ circumstellar dust.

We can use our dust mass estimate in conjunction with the models of N03 and TF01 to understand the dust observed in the early universe. If the progenitor of Cas A was $15 M_{\odot}$, our estimated dust mass $\left(0.020-0.054 M_{\odot}\right)$ is $7 \%-18 \%$ of the $0.3 M_{\odot}$ predicted by the models. If the progenitor mass was $30 M_{\odot}$, then the dust mass is $2 \%-5 \%$ of the $1.1 M_{\odot}$ predicted by the models. One reason our dust mass is lower than that predicted by the models is that we cannot evaluate the mass of very cold dust residing in the remnant from the observed spectra up to $70 \mu \mathrm{m}$ as described above, unless the predicted mass is overestimated. Another reason is that when and how much dust in the remnant is swept up by the reverse shock is highly dependent on the thickness of the hydrogen envelope at the time of explosion and that the evolution and destruction of dust grains formed in $\mathrm{SNe}$ strongly depend not only on their initial sizes but also the density of ambient interstellar medium (Nozawa et al. 2007). Dust formation occurs within a few hundred days after the SN explosion (Kozasa et al. 1989; TF01; N03). Without a thick hydrogen envelope, given an age for Cas A of $\sim 300 \mathrm{yr}$, a significant component of dust may have already been destroyed if dust grains formed in the ejecta were populated by very small-sized grains; otherwise, it is possible that some grain types may be larger, which would increase the inferred mass.

We observed most of the dust compositions predicted by SN Type II models, and the global ejecta composition is consistent with the unmixed-case N03 model rather than the mixed-case model; however, note that different morphologies of $\mathrm{Ar}$ and $\mathrm{Si}$ maps imply that some degree of mixing has occurred. Our estimated dust mass with Spitzer data is 1 order of magnitude smaller than the predicted models of dust formation in SN ejecta by N03 and TF01, but 1-2 orders of magnitude higher than the previous estimations. We now compare the dust mass in highredshift galaxies with the observed dust mass of Cas A based on the chemical evolution model of Morgan \& Edmunds (2003). By a redshift of 4 , SNe have been injecting dust in galaxies for over 2 billion years, and there is enough dust from $\mathrm{SNe}$ to explain the lower limit on the dust masses $\left(\sim 7 \times 10^{7} M_{\odot}\right)$ inferred in submillimeter galaxies and distant quasars (Chini \& Kruegel 1994; Isaak et al. 2002). It should be noted with the dust mass per SN implied by our results for Cas A alone that the interpretation of dust injection from $\mathrm{SNe}$ is limited, because the amount of dust built up over time is strongly dependent on the initial mass function, stellar evolution models, and star formation rates (Morgan \& Edmunds 2003), and destruction rates in supernovae are believed to be important at timescales greater than a few billion years. Additional infrared/submillimeter observations of other young supernova remnants and supernovae are crucial to measure physical processes of dust formation in $\mathrm{SNe}$, including the dust size distribution, composition, and dependence on nucleosynthetic products and environment, and to understand the dust in the early universe in terms of dust injection from $\mathrm{SNe}$.

\section{CONCLUSION}

1. We presented Spitzer IRS mapping covering nearly the entire extent of Cas A and examined if SNe are primary dust formation sites that can be used to explain the high quantity of dust observed in the early universe.

2. The ejecta maps show a remarkable similarity to the dust maps, thereby confirming that dust formation occurs in the SN ejecta.

3. The IRS spectra of Cas A show a few dust features such as a unique $21 \mu \mathrm{m}$ peak in the continuum from $\mathrm{Mg}$ protosilicate, $\mathrm{SiO}_{2}$, and $\mathrm{FeO}$. We observed most of the dust compositions predicted by SN Type II dust models. However, the dust features in Cas A favor $\mathrm{Mg}$ protosilicate rather than $\mathrm{Mg}_{2} \mathrm{SiO}_{4}$, and $\mathrm{FeO}$ rather than $\mathrm{Fe}_{3} \mathrm{O}_{4}$. The composition infers that the ejecta are unmixed.

4. Our total estimated dust mass with Spitzer observations ranging from 5.5 to $70 \mu \mathrm{m}$ is $0.020-0.054 M_{\odot}, 1$ order of magnitude smaller than the predicted models of dust formation in SNe ejecta by N03 and TF01, but 1 or more orders of magnitude higher than the previous estimations. The freshly formed dust mass derived from Cas $\mathrm{A}$ is sufficient from $\mathrm{SNe}$ to explain the lower limit on the dust masses in high-redshift galaxies.

J. R. thanks U. Hwang for helpful discussion of X-ray emission of Cas A. This work is based on observations made with the Spitzer Space Telescope, which is operated by the Jet Propulsion Laboratory, California Institute of Technology, under NASA contract 1407. Partial support for this work was provided by NASA through an GO award issued by JPL/Caltech. 
REFERENCES

Arendt, R. G., Dwek, E., \& Moseley, S. H. 1999, ApJ, 521, 234 (ADM99)

Begemann, B., et al. 1997, ApJ, 476, 199

Bertoldi, F., Carilli, C. L., Cox, P., Fan, X., Strauss, M. A., Beelen, A., Omont, A., \& Zylka, R. 2003, A\&A, 406, L55

Bohren, C. F., \& Huffman, D. R. 1983, Absorption and Scattering of Light by Small Particles (New York: Wiley)

Chevalier, R., \& Soker1989, ApJ, 341, 867

Chini, R., \& Kruegel, E. 1994, A\&A, 288, L33

DeLaney, T. 2004, PhD thesis, Univ. Minnesota

DeLaney, T., Smith, J., Rudnick, L., Ennis, J., Rho, J., Reach, W., Kozasa, T., \& Gomez, H. 2006, AAS Meeting, 208, 59.03

Dorschner, J., Friedmann, C., Gütler, J., \& Duley, W. W. 1980, Ap\&SS, 68, 159

Douvion, T., Lagage, P. O., \& Pantin, E. 2001, A\&A, 369, 589 (D01)

Dunne, L., Eales, S., Ivison, R., Morgan, H., \& Edmunds, M. 2003, Nature, 424, 285

Dwek, E. 2004, ApJ, 607, 848

Dwek, E., Galliano, F., \& Jones, A. P. 2007, ApJ, 662, 927

Dwek, E., Hauser, M. G., Dinerstein, H. L., Gillett, F. C., \& Rice, W. 1987, ApJ, 315, 571

Ennis, J., et al. 2006, ApJ, 652, 376

Ercolano, B., Barlow, M. J., \& Sugerman, B. E. K. 2007, MNRAS, 375, 753

Fesen, R. A. 2001, ApJS, 133, 161

Gomez, H., Dunne, L., Eales, S., Gomez, E., \& Edmunds, M. 2005, MNRAS, 361,1012

Gotthelf, E. V., et al. 2001, ApJ, 552, L39

Hamilton, A. J., \& Fesen, R. 1988, ApJ, 327, 178

Henning, Th., Begemann, B., Mutschke, H., \& Dorschner, J. 1995, A\&AS, 112,143

Hines, D. C., et al. 2004, ApJS, 154, 290

Hirashita, H., et al. 2005, MNRAS, 357, 1077

Hwang, U., Holt, S. S., \& Petre, R. 2000, ApJ, 537, L119

Hwang, U., et al. 2004, ApJ, 615, L117

Isaak, K. G., et al. 2002, MNRAS, 329, 149

Jäger, C., Dorschner, J., Mutschke, H., Posch, Th., \& Henning, Th. 2003, A\&A, 408, 193
Kennicutt, R. C., Jr., et al. 2003, PASP, 115, 928

Kifonidis, K., Plewa, T., Janka, H.-T., \& Müller, E. 2000, ApJ, 531, L123

Kozasa, T., \& Hasegawa, H. 1988, Icarus, 73, 180

Kozasa, T., Hasegawa, H., \& Nomoto, K. 1989, ApJ, 344, 325 . 1991, A\&A, 249, 474

Krause, O., et al. 2004, Nature, 432, 596

Lagage, P. O., et al. 1996, A\&A, 315, L273

Maiolino, R., et al. 2006, Mem. Soc. Astron. Italiana, 77, 643

McCray, R. 1993, ARA\&A, 31, 175

Meikle, W. P. S., et al. 2007, ApJ, 665, 608

Morgan, H. L., Dunne, L., Eales, S. A., Ivison, R. J., \& Edmunds, M. G. 2003, ApJ, 597, L33

Morgan, H. L., \& Edmunds, M. G. 2003, MNRAS, 343, 427

Nozawa, T., Kozasa, T., Umeda, H., Maeda, K., \& Nomoto, K. 2003, ApJ, 598, 785 (N03)

Nozawa, T., et al. 2007, ApJ, 666, 955

Pérez-Rendón, B., García-Segura, G., \& Langer, N. 2002, Rev. Mexicana de Astronomía y Astrofíca, 12, 94

Philipp, H. 1985, in Handbook of Optical Constamts of Solids, ed. E. D. Palik (San Diego: Academic Press), 749

Piller, H. 1985, in Handbook of Optical Constants of Solids, ed. E. D. Palik (London: Academic Press), 571

Reed, J. E., Hester, J. J., Fabian, A. C., \& Winkler, P. F. 1995, ApJ, 440, 706 Rho, J., Reynolds, S. P., Reach, W. T., Jarrett, T. H., Allen, G. E., \& Wilson, J. C. 2003, ApJ, 592, 299

Smith, J. D. T., Aet al. 2007, PASJ, submitted

Sugerman, B. E. K., et al. 2006, Science, 313, 196

Todini, P., \& Ferrara, A. 2001, MNRAS, 325, 726 (TF01)

van den Bergh, S. 1971, ApJ, 165, 457

Wilson, T. L., \& Batrla, W. 2005, A\&A, 430, 561

Woosley, S. E., A. Heger, A., \& Weaver, T. A 2002, Rev. Mod. Phys., 74, 1015

Woosley, S. E., \& Weaver, T. A. 1995, ApJS, 101, 181

Young, P. A., et al. 2006, ApJ, 640, 891 